\title{
Manejo da solução nutritiva sobre a qualidade pós-colheita de tomate salada fertirrigado em areia
}

\author{
Management of the nutrient solution for postharvest quality of tomatoes with \\ fertigation in sand
}

\author{
Hideaki Wilson Takahashi', Renan Ribeiro Barzan', Douglas Junior Bertoncelli', Ana Beatryz Prenzier Suzuki', \\ Gustavo Adolfo de Freitas Fregonezi ${ }^{2}$, Marita Di Loreto Y Sampaio', Ruan Francisco Firmano ${ }^{3}$, \\ Josemeyre Bonifácio da Silva ${ }^{1 *}$ \\ 1 Universidade Estadual de Londrina (UEL), Centro de Ciências Agrárias, Departamento de Agronomia, Campus Universitário, Londrina/PR - Brasil \\ ${ }^{2}$ Centro Universitário Filadélfia (UNIFIL), Departamento de Agronomia, Campus Canadá, Londrina/PR - Brasil \\ ${ }^{3}$ Universidade de São Paulo (USP), Escola Superior de Agricultura "Luiz de Queiroz" (ESALQ), Piracicaba/SP - Brasil
}

\section{*Corresponding Author}

Josemeyre Bonifácio da Silva, Universidade Estadual de Londrina (UEL), Centro de Ciências Agrárias, Departamento de Agronomia, Rodovia Celso Garcia Cid, PR 445, km 380, Campus Universitário, CEP: 86057-970, Londrina/PR - Brasil, e-mail: josibonifacio@uel.br

Cite as: Management of the nutrient solution for postharvest quality of tomatoes with fertigation in sand. Braz. J. Food Technol., v. 21, e2016144, 2018.

Received: Dec. 10, 2016; Accepted: Aug. 14, 2017

\section{Resumo}

O presente trabalho teve como objetivo avaliar o efeito de manejos e concentrações da solução nutritiva em fertirrigação, em cultivo protegido, sobre a qualidade pós-colheita de tomate de mesa. Os tratamentos consistiram do manejo da solução nutritiva, tendo como base a condutividade elétrica (CE) e a concentração individual de íons (Cl) do lixiviado, nas concentrações iniciais de 1,6 e 3,2 dS $\mathrm{m}^{-1}$. Os frutos foram avaliados quanto aos teores de macrominerais, sólidos solúveis totais (SST), vitamina C, compostos fenólicos totais, flavonoides totais e compostos antioxidantes. Os teores de N, P e S foram maiores no manejo da solução nutritiva com base na $\mathrm{Cl}$, independentemente da concentração inicial de íons. Os teores de $\mathrm{K}$ e $\mathrm{Ca}$ apresentaram efeitos da interação entre a forma de manejo e a concentração de íons, enquanto o teor de $\mathrm{Mg}$ não foi influenciado pelos fatores estudados. O teor de vitamina $\mathrm{C}$ e a atividade antioxidante foram maiores no manejo da solução com base em CE, enquanto o teor de fenóis e flavonoides foi maior no manejo da solução com base em Cl. O tipo de manejo e a concentração da solução nutritiva em fertirrigação afetam a qualidade pós-colheita de frutos de tomate.

Palavras-chave: Lycopersicon esculentum Mill.; Fertirrigação; Minerais; Fenóis; Flavonoides; Antioxidantes.

\section{Abstract}

The aim of this study was to evaluate the effect of the management of the nutrient solution concentrations used in the fertigation of table tomatoes cultivated in a protected environment, on postharvest quality. The treatments consisted of managing the nutrient solution based on the electrical conductivity (EC) and individual ionic concentrations (IC) in the leached solution at the levels of 1.6 and $3.2 \mathrm{dS} \mathrm{m}^{-1}$. The fruits were evaluated for their macromineral contents, total soluble solids (TSS), vitamin C, total phenols, flavonoids and antioxidants. The N, P and S contents were higher for management of the nutrient solution based on the IC, regardless of the initial ion concentrations. The $\mathrm{K}$ and $\mathrm{Ca}$ contents showed interaction effects between the forms of management and ion concentrations, while the Mg content was not influenced by the factors studied. The vitamin $\mathrm{C}$ content and antioxidant activity were higher for management of the solution based on EC, while total phenols and flavonoids were greater for management based on IC. The type of management and the nutrient solution concentration used in fertigation affect the postharvest quality of tomato fruits.

Keywords: Lycopersicon esculentum Mill.; Fertigation; Minerals; Phenols; Flavonoids; Antioxidants. 


\section{Introdução}

O tomateiro (Lycopersicon esculentum Mill.) é uma das hortaliças mais consumidas no mundo, com uma produção mundial de 163 milhões de toneladas, em 2013. Deste montante, aproximadamente $20 \%$ concentram-se nas Américas (FAOSTAT, 2013). A composição química dos frutos é influenciada pelos seguintes fatores: cultivar, estádio de maturação, clima, condições de cultivo, processamento e armazenamento (MORITZ; TRAMONTE, 2006).

Os frutos in natura apresentam 1,32\% de proteínas, 0,39\% de lipídeos, 7,09\% de carboidratos e 1,4\% de fibras. $O$ tomate ainda é considerado fonte de vitaminas ( $C$, tiamina e outras), minerais (K, Mg e outros) e substâncias com atividades antioxidantes, como carotenoides, flavonoides e compostos fenólicos (BORGUINI; TORRES, 2009). Com isto, seu consumo tem sido associado à redução de câncer de próstata e de doenças crônicas degenerativas (VIDAL et al., 2012).

A cultura do tomateiro destinada à mesa apresenta grande diversidade de sistemas de produção, com destaque para o cultivo protegido associado à fertirrigação em substratos, visando a maior aproveitamento dos nutrientes, produtividade e qualidade dos frutos (CARRIJO et al., 2004; FONTES et al., 2004). Ainda, diferentes formas de manejar o fornecimento de nutrientes por meio de solução nutritiva podem ser adotadas no cultivo do tomateiro fertirrigado, influenciando o estado nutricional das plantas e, logo, a composição química e a qualidade do produto colhido.

Uma das técnicas adotadas no manejo da fertirrigação é o monitoramento do lixiviado ou Pour-Through (CAVINS et al., 2008), cujos diferentes atributos podem ser avaliados, como a condutividade elétrica (CE) e as concentrações de diferentes íons. A CE resulta da presença dos íons em solução e é diretamente relacionada com a concentração destes (HELBEL JUNIOR et al., 2008). É uma forma prática, porém menos detalhada do que as concentrações iônicas, de obter informações sobre o balanço entre fornecimento e demanda de nutrientes pelas plantas.

Poucos trabalhos descrevem a influência da fertirrigação na qualidade pós-colheita de frutos de tomateiro. Macedo e Alvarenga (2005) avaliaram o efeito das lâminas de água e da fertirrigação potássica sobre a qualidade de tomate em ambiente protegido, e observaram que o teor de sólidos solúveis aumentou em função das doses de K. Chapagain e Wiesman (2004) concluíram que o uso de
$\mathrm{KCl} \cdot \mathrm{MgCl}_{2}$ na fertirrigação aumentou o nível de Mg foliar e a qualidade geral dos frutos de tomate.

Sampaio e Fontes (1998) relataram que a aplicação de K por fertirrigação aumentou o teor de Ca no fruto, sendo que as relações $\mathrm{K} / \mathrm{Mg}$ e $\mathrm{K} /(\mathrm{Ca}+\mathrm{Mg})$ no fruto foram, em geral, menores no tratamento com fertirrigação total. Diversamente, a percentagem de sólidos solúveis totais, a acidez total titulável, o "flavor", o pH e o teor de vitamina $C$, no fruto de tomate, não foram influenciados pela fertirrigação.

Portanto, o presente trabalho teve como objetivo avaliar o efeito de manejos e concentrações da solução nutritiva em fertirrigação, em cultivo protegido, sobre a qualidade pós-colheita de tomate de mesa.

\section{Material e métodos}

O experimento foi conduzido em casa de vegetação (modelo 'teto em arco'), no Centro de Ciências Agrárias da Universidade Estadual de Londrina (UEL), Londrina-PR (Latitude: $23^{\circ} 23^{\prime} \mathrm{S}$; Longitude: 51 $11^{\prime} \mathrm{W}$; Altitude: 560 m). O cultivo foi feito em sistema de fertirrigação por gotejamento, usando-se vasos de polipropileno contendo areia grossa como substrato.

O delineamento experimental foi em blocos casualizados, em esquema fatorial $2 \times 2$, com 5 repetições. $O$ primeiro fator consistiu da forma de manejo da solução nutritiva com base no lixiviado, utilizando-se a condutividade elétrica (CE) ou as concentrações de íons (Cl), como critérios. O segundo fator foi o nível de concentração de nutrientes inicial, representado pelas condutividades elétricas de 1,6 e 3,2 dS m-1, na solução nutritiva (Tabela 1).

O híbrido de tomateiro utilizado foi o Paronset, tipo salada, de crescimento indeterminado. As mudas foram obtidas em viveiro certificado, sendo transplantadas no dia 01 de maio de 2014. O espaçamento foi de 0,8 m entre linhas e 0,3 m entre vasos na linha (41.666 plantas ha $^{-1}$ ) e cada vaso, contendo uma planta de tomateiro, foi considerado como uma unidade experimental. Nos primeiros 15 dias após o transplantio (DAT), foi utilizada uma solução nutritiva de adaptação, com condutividade de 1,2 dS m-1,

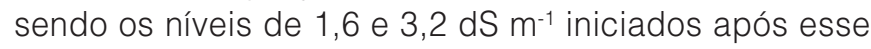
período. A coleta de lixiviado (20 $\mathrm{mL}$ vaso $\left.^{-1}\right)$ para manejo da solução nutritiva teve início aos 56 DAT e foi realizada semanalmente, uma hora após a fertirrigação.

A condutividade elétrica (CE) foi medida em condutivímetro portátil e as concentrações dos íons

Tabela 1. Concentração inicial de nutrientes das soluções nutritivas em dois níveis de condutividade elétrica (CE), utilizados na fertirrigação do tomateiro.

\begin{tabular}{|c|c|c|c|c|c|c|c|c|c|c|c|c|}
\hline Nutriente & $\mathbf{N}$ & $\mathbf{P}$ & K & $\mathrm{Ca}$ & $\mathrm{Mg}$ & $S$ & B & $\mathrm{Cu}$ & $\mathrm{Fe}$ & $\mathrm{Zn}$ & Mn & Mo \\
\hline CE & & & 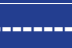 & - & -+ & $-n$ & $-1 \ldots$ & - & 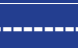 & ----S-S & & \\
\hline $1,6 \mathrm{dS} \mathrm{m}^{-1}$ & 198 & 43,6 & 152 & 233 & 27 & 39 & 0,63 & 0,11 & 2,75 & 1,95 & 0,74 & 0,01 \\
\hline $3,2 \mathrm{dS} \mathrm{m}^{-1}$ & 396 & 87,2 & 304 & 466 & 54 & 78 & 1,26 & 0,22 & 5,50 & 3,90 & 1,48 & 0,02 \\
\hline
\end{tabular}


N, P, K, Ca, Mg e S do lixiviado foram obtidas de acordo com as metodologias descritas por Silva (2009). No manejo CE, a solução nutritiva, como um todo, era diluída ou concentrada em $20 \%$, quando o valor da condutividade elétrica do lixiviado se encontrava, respectivamente, acima ou abaixo da faixa pré-estabelecida (aproximadamente $20 \%$, em relação às respectivas concentrações iniciais) (Tabela 1). Para o manejo $\mathrm{Cl}$, o mesmo procedimento foi adotado, porém, corrigindo-se separadamente a concentração de cada íon da solução nutritiva, em função do respectivo valor obtido no lixiviado.

Uma parte dos frutos colhidos manualmente, conforme atingiram a maturação, foi avaliada quanto ao teor de sólidos solúveis totais (SST), por leitura do suco da polpa em refratômetro portátil (marca Atago, modelo PAL). Os resultados, média de três repetições, foram expressos em ${ }^{\circ}$ Brix. Outra parte, frutos com casca e semente, foi seca em estufa de ventilação de ar à temperatura de $55^{\circ} \mathrm{C}$, sendo os frutos triturados em moinho tipo Wiley e armazenados em câmara fria, para posteriores análises químicas.

Todos os reagentes utilizados foram de grau analítico e de diferentes procedências.

Os teores dos macrominerais foram determinados conforme metodologias descritas por Silva (2009). No extrato da digestão nitroperclórica, foram quantificados fósforo $(P)$ e enxofre (S), por colorimetria; cálcio (Ca) e magnésio (Mg), por espectrofotometria de absorção atômica, e potássio $(\mathrm{K})$, por fotometria de chama. O teor de nitrogênio $(\mathrm{N})$ foi obtido mediante digestão sulfúrica e quantificação pelo método de Kjeldahl (IAL, 2008). Os resultados, média de três repetições, foram expressos em $\mathrm{g} \mathrm{kg}^{-1}$

O teor de vitamina $\mathrm{C}$ foi determinado pelo método Tillmans (IAL, 2008). Em $10 \mathrm{~g}$ de amostra fresca, foram adicionados $50 \mathrm{~mL}$ de $\mathrm{H}_{2} \mathrm{O}$ destilada e $50 \mathrm{~mL}$ de ácido oxálico a 1\%. Em seguida, procedeu-se à titulação com 2,6-diclorofenol indofenol até a obtenção de coloração rósea persistente. Os resultados, foram expressos em mg $100 \mathrm{~g}^{-1}$.

Os compostos fenólicos totais foram quantificados por espectrofotometria, de acordo com Stratil et al. (2006), com algumas modificações. Para a extração, $1 \mathrm{~g}$ de amostra foi colocada em Erlenmeyer de $250 \mathrm{~mL}$ com $10 \mathrm{~mL}$ de etanol e, logo em seguida, os frascos foram colocados em mesa agitadora durante 30 min a 2.500 rpm, à temperatura ambiente $\left(25^{\circ} \mathrm{C}\right)$. Após a agitação, os sobrenadantes foram coletados em tubos de centrífuga do tipo Falcon (50 mL) e centrifugados (Centrilab TDZ5) por 5 min a $2.500 \mathrm{rpm}$, à temperatura ambiente $\left(25^{\circ} \mathrm{C}\right)$. Os sobrenadantes foram utilizados para análise. Em tubos de ensaio, foram adicionados: 0,05 $\mathrm{mL}$ do sobrenadante, $2 \mathrm{~mL}$ de Folin-Ciocalteu (Sigma) 0,9 N, 2,0 mL de $\mathrm{H}_{2} \mathrm{O}$ destilada, $1 \mathrm{~mL}$ de solução de carbonato de sódio $\left(\mathrm{Na}_{2} \mathrm{CO}_{3}\right)$, sendo, então, homogeneizados. Em seguida, os tubos de ensaio foram mantidos em repouso e ao abrigo da luz durante 30 min, para o desenvolvimento da cor, procedendo-se, posteriormente, à leitura da absorbância a 720 nm, em espectrofotômetro (Micronal, AJX-1600). Para a quantificação dos fenólicos totais, foi construída uma curva de calibração usando-se diferentes concentrações do padrão $\left(1,5,10,20,40,60\right.$ e $80 \mu \mathrm{g} \mathrm{mL} \mathrm{m}^{-1}$ de ácido gálico). A partir da equação da reta obtida, foi realizado o cálculo de fenólicos totais cujos resultados, média de três repetições, foram expressos em mg $100 \mathrm{~g}^{-1}$.

A extração de flavonoides seguiu o mesmo procedimento dos compostos fenólicos até a obtenção do sobrenadante; a análise foi realizada conforme Woisky e Salatino (1998), com algumas modificações. Em tubos de ensaio, foram adicionados $2 \mathrm{~mL}$ do extrato etanólico (sobrenadante), $2 \mathrm{~mL}$ de cloreto de alumínio 5\% (m/v) e $2 \mathrm{~mL}$ de metanol. Para a reação, as amostras foram deixadas em repouso durante $30 \mathrm{~min}$, no escuro, e procedeu-se à leitura em espectrofotômetro (Micronal, AJX-1600) a $425 \mathrm{~nm}$. A quercetina foi utilizada nas concentrações de 50, 100, 200, 300, 400 e $500 \mathrm{mg} \mathrm{mL}^{-1}$, para a construção da curva de calibração. A partir da equação da reta obtida, foi realizado o cálculo do teor de flavonoides, sendo os resultados, média de três repetições, expressos em $\mathrm{mg} \mathrm{g}^{-1}$.

$A$ atividade antioxidante foi determinada conforme metodologia descrita por Aruoma (2003). Para a extração, $1 \mathrm{~g}$ de amostra foi colocado em tubos de centrífuga do tipo Falcon (50 mL), adicionando-se $20 \mathrm{~mL}$ de metanol $50 \%$. As amostras foram deixadas em repouso, durante $30 \mathrm{~min}$, à temperatura ambiente $\left(25^{\circ} \mathrm{C}\right)$. Logo em seguida, foram centrifugadas (Centrilab, TDZ5) a 25.000 rpm por $5 \mathrm{~min}$, transferindo-se o sobrenadante para novos tubos. No sobrenadante da primeira extração, foram adicionados $20 \mathrm{~mL}$ de acetona $70 \%$. Novamente, os tubos foram homogeneizados e deixados em repouso por mais 30 min, à temperatura ambiente $\left(25^{\circ} \mathrm{C}\right)$. Procedeu-se à centrifugação, nas mesmas condições supradescritas, e o sobrenadante foi usado para análise. Em tubos de ensaio, transferiram-se alíquotas de 0,1 mL de cada diluição do extrato com 3,9 mL do radical 2,2-difenil-1-picrilhidrazila (DPPH). Foi utilizada a solução com álcool metílico e DPPH como controle, bem como o metanol para calibrar o espectrofotômetro (Micronal, AJX-1600). As leituras foram realizadas após 30 min e a $515 \mathrm{~nm}$. Para a quantificação, foi construída a curva padrão com o DPPH em diferentes concentrações $(0,10,20,30,40,50$ e $60 \mu \mathrm{M})$ e os resultados, média de três repetições, foram expressos em $\mathrm{mg} \mathrm{g}^{-1}$ de DDPH.

Os dados foram testados quanto a normalidade e homocedasticidade dos resíduos, pelos testes de Shapiro Wilk e de Hartley, respectivamente, sendo então submetidos à análise de variância (ANOVA) e à comparação de médias pelo teste $t$ de Student a $5 \%$ de probabilidade de erro, utilizando-se o programa Sisvar (FERREIRA, 2011). 


\section{Resultados e discussão}

Os maiores teores de N, P e S, independentemente da concentração inicial da solução nutritiva, foram observados no manejo com base nas concentrações dos íons (Cl) do lixiviado (Tabela 2). Para N e S, o aumento na concentração de 1,6 para 3,2 dS $\mathrm{m}^{-1}$, nas duas formas de manejo, também aumentou seus teores nos frutos de tomate.

O teor de Mg não teve efeito dos fatores estudados, enquanto que para $\mathrm{K}$ e Ca, houve interação. A associação do manejo CE com a concentração de 1,6 dS m-1 proporcionou valores inferiores para $\mathrm{K}$ e superiores para Ca. Segundo Malavolta (2006), estes dois nutrientes apresentam inibição competitiva no processo de absorção nas raízes, o que reflete na quantidade em que ambos são transportados e alocados nos frutos.

Os teores de vitamina $\mathrm{C}$, antioxidantes, fenóis e flavonoides tiveram efeitos isolados do manejo e das concentrações da solução nutritiva, enquanto o teor de sólidos solúveis apresentou efeito da interação (Tabela 3).

O teor de vitamina C e a atividade antioxidante foram maiores no manejo da solução nutritiva com base na CE; já para fenóis totais e flavonoides, os maiores teores foram obtidos no manejo da solução nutritiva com base na $\mathrm{Cl}$. A concentração inicial de 3,2 dS $\mathrm{m}^{-1}$ proporcionou maiores teores de fenóis totais e flavonoides, ao passo que a vitamina $\mathrm{C}$ foi reduzida.
Os frutos do tomateiro apresentaram maior capacidade antioxidante quando manejados em solução nutritiva com base na CE do lixiviado, sem apresentar efeito para as concentrações iniciais. Para o teor de sólidos solúveis totais, foi observado menor valor em função do manejo pela condutividade elétrica do lixiviado, na concentração de $1,6 \mathrm{dS} \mathrm{m} \mathrm{m}^{-1}$.

Os resultados obtidos comprovam a importância do manejo da nutrição mineral dos frutos de tomate sobre a qualidade pós-colheita. Isto pode ser observado, por exemplo, pelo fato de que os tratamentos de maior alocação de N,P e S nos frutos terem sido também aqueles em que houve maior concentração de fenóis e flavonoides. As classes mais abundantes de fenóis em plantas são derivadas da rota do ácido chiquímico, que tem como precursores metabólitos primários, como o ácido fosfoenolpirúvico e a eritrose-4-P, além de aminoácidos de cadeia aromática (TAIZ; ZEIGER, 2013).

Como N, P e S apresentam papel fundamental em reações metabólicas, as quais dão origem aos precursores de compostos fenólicos, é compreensível o aumento destes em função da maior concentração dos referidos nutrientes. Ainda, uma vez que os flavonoides constituem grande parte dos compostos fenólicos vegetais, a elevação dos valores de fenóis totais está diretamente relacionada ao aumento nos níveis de flavonoides, especialmente a quercetina, principal flavonoide presente no tomate (MONTEIRO, 2008).

Tabela 2. Teores de macrominerais nos frutos de tomateiro submetido a diferentes manejos e concentrações da solução nutritiva em cultivo fertirrigado.

\begin{tabular}{|c|c|c|c|c|c|}
\hline \multirow{2}{*}{ Nutriente $\left(\mathrm{g} \mathrm{kg}^{-1}\right)$} & \multirow{2}{*}{ Forma de manejo ${ }^{(1)}$} & \multicolumn{2}{|c|}{ Concentração (dS $\mathrm{m}^{-1}$ ) } & \multirow{2}{*}{ Média } & \multirow{2}{*}{ C.V. (\%) } \\
\hline & & 1,6 & 3,2 & & \\
\hline \multirow{3}{*}{ Nitrogênio (N) } & CE & 21,01 & 24,02 & $22,51^{\mathrm{B}}$ & \multirow{3}{*}{8,07} \\
\hline & $\mathrm{Cl}$ & 25,44 & 30,03 & $27,74^{\mathrm{A}}$ & \\
\hline & Média & $23,22^{b}$ & $27,03^{a}$ & - & \\
\hline \multirow{3}{*}{ Fósforo (P) } & CE & 2,49 & 2,56 & $2,53^{B}$ & \multirow{3}{*}{15,42} \\
\hline & $\mathrm{Cl}$ & 3,22 & 3,33 & $3,27^{A}$ & \\
\hline & Média & $2,85^{a}$ & $2,95^{a}$ & - & \\
\hline \multirow{3}{*}{ Potássio (K) } & CE & 29,03 bв & 35,13 aA & 32,08 & \multirow{3}{*}{9,60} \\
\hline & $\mathrm{Cl}$ & 40,70 aA & 39,30 aA & 40,00 & \\
\hline & Média & 34,87 & 37,22 & - & \\
\hline \multirow{3}{*}{ Cálcio (Ca) } & CE & $8,04^{\mathrm{aA}}$ & $5,76^{\mathrm{bA}}$ & 6,90 & \multirow{3}{*}{20,13} \\
\hline & $\mathrm{Cl}$ & $4,01 \mathrm{aB}$ & $4,044^{a B}$ & 4,02 & \\
\hline & Média & 6,03 & 4,90 & - & \\
\hline \multirow{3}{*}{ Magnésio (Mg) } & CE & 0,98 & 0,89 & $0,93^{A}$ & \multirow{3}{*}{11,05} \\
\hline & $\mathrm{Cl}$ & 0,86 & 0,87 & $0,87^{A}$ & \\
\hline & Média & $0,92^{a}$ & $0,88^{a}$ & - & \\
\hline \multirow{3}{*}{ Enxofre (S) } & CE & 1,49 & 1,76 & $1,63^{\mathrm{B}}$ & \multirow{3}{*}{15,85} \\
\hline & $\mathrm{Cl}$ & 1,80 & 2,62 & $2,21^{\mathrm{A}}$ & \\
\hline & Média & $1,65^{b}$ & $2,19^{a}$ & - & \\
\hline
\end{tabular}

Médias seguidas por letras distintas, maiúsculas entre as formas de manejo da solução nutritiva e minúsculas entre as concentrações inicias, diferem entre si pelo teste $t$ de Student no nível de 5\%; (1) CE = Manejo da solução nutritiva com base na condutividade elétrica do lixiviado; $\mathrm{Cl}=$ Manejo da solução nutritiva com base nas concentrações dos íons do lixiviado. 
Manejo da solução nutritiva sobre a qualidade pós-colheita de tomate salada fertirrigado em areia

Takahashi, H. W. et al.

Tabela 3. Teores de sólidos solúveis totais (SST), vitamina C, fenóis totais, flavonoides e antioxidantes dos frutos de tomateiro submetido a diferentes manejos e concentrações da solução nutritiva em cultivo fertirrigado.

\begin{tabular}{|c|c|c|c|c|c|}
\hline \multirow{2}{*}{ Característica } & \multirow{2}{*}{$\begin{array}{l}\text { Forma de } \\
\text { manejo(1) }\end{array}$} & \multicolumn{2}{|c|}{ Concentração (dS m ${ }^{-1}$ ) } & \multirow{2}{*}{ Média } & \multirow{2}{*}{ C.V. $(\%)$} \\
\hline & & 1,6 & 3,2 & & \\
\hline \multirow{3}{*}{ SST ( ${ }^{\circ}$ Brix) } & CE & $4,23 \mathrm{bB}$ & $4,83^{\text {aA }}$ & 4,53 & \multirow{3}{*}{9,79} \\
\hline & $\mathrm{Cl}$ & 4,77 aA & 4,71 aA & 4,74 & \\
\hline & Média & 4,50 & 4,77 & - & \\
\hline \multirow{3}{*}{ Vitamina $C\left(\mathrm{mg} 100 \mathrm{~g}^{-1}\right)$} & CE & 18,68 & 17,36 & $18,02^{A}$ & \multirow{3}{*}{7,14} \\
\hline & $\mathrm{Cl}$ & 15,60 & 14,54 & $15,07^{B}$ & \\
\hline & Média & $17,14^{a}$ & $15,95^{b}$ & - & \\
\hline \multirow{3}{*}{ Fenóis Totais (mg $\left.100 \mathrm{~g}^{-1}\right)$} & CE & 240,88 & 249,66 & $245,27 \mathrm{~B}$ & \multirow{3}{*}{0,40} \\
\hline & $\mathrm{Cl}$ & 257,16 & 281,13 & $269,15^{A}$ & \\
\hline & Média & 249,02 b & 265,40 a & - & \\
\hline \multirow{3}{*}{ Flavonoides (mg EQ g ${ }^{-1}$ ) } & CE & 32,99 & 43,65 & $38,32^{\mathrm{B}}$ & \multirow{3}{*}{1,34} \\
\hline & $\mathrm{Cl}$ & 50,44 & 59,40 & $54,92^{A}$ & \\
\hline & Média & $41,72^{b}$ & $51,53^{a}$ & - & \\
\hline \multirow{3}{*}{ Antioxidantes (mg DPPH g $^{-1}$ ) } & CE & 3,95 & 4,12 & $4,03^{A}$ & \multirow{3}{*}{8,42} \\
\hline & $\mathrm{Cl}$ & 3,70 & 3,70 & $3,70^{\mathrm{B}}$ & \\
\hline & Média & $3,82^{a}$ & $3,91^{\text {a }}$ & - & \\
\hline
\end{tabular}

Médias seguidas por letras distintas, maiúsculas entre as formas de manejo da solução nutritiva e minúsculas entre as concentraç̃es inicias, diferem entre si pelo teste $t$ de Student no nível de $5 \%$; (1) CE = Manejo da solução nutritiva com base na condutividade elétrica do lixiviado; $\mathrm{Cl}=$ Manejo da solução nutritiva com base nas concentrações dos íons do lixiviado.

O efeito do manejo e da concentração da solução nutritiva sobre a alocação de nutrientes nos frutos - e, em consequência, alterando outros compostos - é evidenciado também pela relação obtida entre os sólidos solúveis totais (SST) e o teor de K. Sabe-se que o K é um nutriente que atua na relação fonte-dreno, no que diz respeito ao transporte de fotoassimilados (MARSCHNER, 2011).

Dessa forma, o menor teor de SST nos frutos, obtido pelo manejo da solução nutritiva com base na CE do lixiviado no nível de 1,6 dS $\mathrm{m}^{-1}$, pode ser explicado, em parte, pela menor disponibilidade e pelo transporte de K. Fratoni (2014) relatou um incremento linear no teor de SST em frutos de tomateiro, em função de doses crescentes de K na solução nutritiva. Apesar disso, mesmo o menor valor obtido de SST está dentro da faixa normalmente encontrada para tomate de mesa (FERREIRA, 2004).

Os valores de vitamina $C$ se apresentaram abaixo do valor médio de $34,3 \mathrm{mg} 100 \mathrm{~g}^{-1}$, indicado para tomate nacional (LUENGO, 2000). Contudo, Sousa et al. (2011) relataram valores entre 7,20 e 45,60 mg $100 \mathrm{~g}^{-1}$, que podem ser encontrados, dependendo de determinados aspectos, como época do ano, luz, adubação e substrato. Os maiores

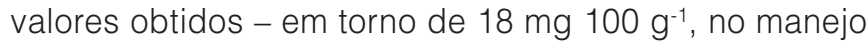
CE, e no nível de 1,6 dS $\mathrm{m}^{-1}$ - podem ser explicados pela menor alocação de nitrogênio nos frutos. Lee e Kader (2000) relataram que os teores de vitamina $C$ tendem a ser reduzidos com o aumento na disponibilidade de $\mathrm{N}$.

Diversos são os compostos com atividade antioxidante em tomate, incluindo os próprios compostos fenólicos e a vitamina $\mathrm{C}$, além de carotenoides, como o $\beta$-caroteno e, especialmente, o licopeno; contudo, a contribuição de cada um para a capacidade antioxidante total ainda é pouco conhecida (ZAPATA et al., 2007). No presente estudo, pode-se evidenciar uma maior relação entre a capacidade antioxidante e a concentração de vitamina $\mathrm{C}$, que foram ambas superiores no manejo CE.

\section{Conclusões}

A forma de manejo e a concentração inicial da solução nutritiva em fertirrigação afetam a qualidade pós-colheita de frutos de tomate. A resposta a estes fatores varia conforme os componentes da qualidade dos frutos.

Os maiores teores de cálcio e vitamina C, e a maior atividade antioxidante são obtidos no manejo com base na condutividade elétrica do lixiviado e concentração de 1,6 dS m-1.

Os compostos fenólicos e minerais relacionados a proteínas (nitrogênio e enxofre), têm seus teores elevados com o manejo pelas concentrações individuais de íons e concentração de $3,2 \mathrm{dS} \mathrm{m}^{-1}$.

\section{Referências}

ARUOMA, O. I. Methodological considerations for characterizing potential antioxidant actions of bioactive components in plant foods. Mutation Research, v. 523-524, p. 9-20, 2003. PMid:12628499. http://dx.doi.org/10.1016/S0027-5107(02)00317-2.

BORGUINI, R. G.; TORRES, E. A. F. D. Tomatoes and tomato products as dietary sources of antioxidants. Food Research International, v. 25, n. 4, p. 313-325, 2009. http://dx.doi. org/10.1080/87559120903155859. 
Manejo da solução nutritiva sobre a qualidade pós-colheita de tomate salada fertirrigado em areia Takahashi, H. W. et al.

CARRIJO, O. A.; VIDAL, M. C.; REIS, N. V. B.; SOUZA, R. B.; MAKISHIMA, N. Produtividade do tomateiro em diferentes substratos e modelos de casas de vegetação. Horticultura Brasileira, v. 22, n. 1, p. 5-9, 2004. http://dx.doi.org/10.1590/ S0102-05362004000100001.

CAVINS, T. J.; WHIPKER, B. E.; FONTENO, W. C. PourThru: a method for monitoring nutrition in the greenhouse. Acta Horticulturae, n. 779, p. 289-297, 2008. http://dx.doi.org/10.17660/ ActaHortic.2008.779.35.

CHAPAGAIN, B. P.; WIESMAN, Z. Effect of potassium magnesium chloride in the fertigation solution as partial source of potassium on growth, yield and quality of greenhouse tomato. Scientia Horticulturae, v. 99, n. 3-4, p. 279-288, 2004. http://dx.doi. org/10.1016/S0304-4238(03)00109-2.

FERREIRA, D. F. Sisvar: a computer statistical analysis system. Ciência e Agrotecnologia, v. 35, n. 6, p. 1039-1042, 2011. http:// dx.doi.org/10.1590/S1413-70542011000600001.

FERREIRA, S. M. R. Características de qualidade do tomate de mesa (Lycopersicon esculentum Mill.) cultivado nos sistemas convencional e orgânico comercializado na região metropolitana de Curitiba. 2004. 231 f. Tese (Doutorado em Tecnologia de Alimentos)-Universidade Federal do Paraná, Curitiba, 2004

FONTES, P. C. R.; LOURES, J. L.; GALVÃO, J. C.; CARDOSO, A. A.; MANTOVANI, E. C. Produção e qualidade do tomate produzido em substrato, no campo e em ambiente protegido. Horticultura Brasileira, v. 22, n. 3, p. 614-619, 2004. http:// dx.doi.org/10.1590/S0102-05362004000300023.

FOOD AND AGRICULTURE ORGANIZATION OF THE UNITED NATIONS - FAOSTAT. Production/crops: tomatoes, World. Rome: FAOSTAT, 2013. Disponível em: <http://faostat3.fao.org/ browse/Q/QC/E>. Acesso em: 8 mar. 2016.

FRATONI, M. M. J. Nutrição potássica em tomateiro fertirrigado e cultivado em vasos contendo areia. 2014. 53 f. Dissertação (Mestrado)-Universidade Estadual de Londrina, Londrina, 2014.

HELBEL JUNIOR, C.; REZENDE, R.; FREITAS, P. S. L.; GONÇALVES, A. C. A.; FRIZZONE, J. A. Influência da condutividade elétrica, concentração iônica e vazão de soluções nutritivas na produção de alface hidropônica. Ciência e Agrotecnologia, v. 32, n. 4, p. 1142-1147, 2008. http://dx.doi.org/10.1590/S141370542008000400016 .

INSTITUTO ADOLFO LUTZ - IAL. Normas analíticas do Instituto Adolfo Lutz: métodos físico-químicos para análise de alimentos. 4. ed. São Paulo: IAL, 2008. 1020 p.

LEE, S. K.; KADER, A. A. Preharvest and postharvest factors influencing vitamin $\mathrm{C}$ content of horticultural crops. Postharvest Biology and Technology, v. 20, n. 3, p. 207-220, 2000. http:// dx.doi.org/10.1016/S0925-5214(00)00133-2.
LUENGO, R. F. A. Tabela de composição nutricional das hortaliças Brasília: EMBRAPA Hortaliças, 2000. 4 p. (Documentos; 26).

MACEDO, L. S.; ALVARENGA, M. A. R. Efeitos de lâminas de água e fertirrigação potássica sobre o crescimento, produção e qualidade do tomate em ambiente protegido. Ciência e Agrotecnologia, v. 29, n. 2, p. 296-304, 2005. http://dx.doi. org/10.1590/S1413-70542005000200005.

MALAVOLTA, E. Manual de nutrição mineral de plantas. Ouro Fino: Agronômica Ceres, 2006. 632 p.

MARSCHNER, P. Marschner's mineral nutrition of higher plants. 3rd ed. Waltham: Academic Press. 672 p. 2011

MONTEIRO, C. S. Desenvolvimento de molho de tomate Lycopersicon esculentum Mill formulado com cogumelo Agaricus brasiliensis. 2008. $174 \mathrm{f}$. Tese (Doutorado em Tecnologia de Alimentos)-Universidade Federal do Paraná, Curitiba, 2008.

MORITZ, B.; TRAMONTE, V. L. C. Biodisponibilidade do licopeno. Revista de Nutrição, v. 19, n. 2, p. 265-273, 2006. http://dx.doi. org/10.1590/S1415-52732006000200013.

SAMPAIO, R. A.; FONTES, P. C. Qualidade de frutos de tomateiro fertirrigado com potássio em solo coberto com polietileno preto. Horticultura Brasileira, v. 16, n. 2, p. 136-139, 1998. http:// dx.doi.org/10.1590/S0102-05361998160000200009. </jrn>x

SILVA, F. C. Manual de análises químicas de solos, plantas e fertilizantes. 2. ed. Brasília: Embrapa Informação Tecnológica, 2009. $627 \mathrm{p}$.

SOUSA, A. A.; GRIGIO, M. L.; NASCIMENTO, C. R.; SILVA, A. C. D.; REGO, E. R.; REGO, M. M. Caracterização química e física de frutos de diferentes acessos de tomateiro em casa de vegetação. Revista Agroambiente, v. 5, p. 113-118, 2011.

STRATIL, P.; KLEJDUS, B.; KUBÁN, V. Determination of total content of phenolic compounds and their antioxidant activity in vegetables-evaluation of spectrophotometric methods. Journal of Agricultural and Food Chemistry, v. 54, n. 3, p. 607-616, 2006. PMid:16448157. http://dx.doi.org/10.1021/jf052334j.

TAIZ, L.; ZEIGER, E. Fisiologia vegetal. 5. ed. Porto Alegre: Artmed, 2013. 954 p.

VIDAL, M. A.; DIAS, D. O.; MARTINS, E. S. M.; OLIVEIRA, R. S.; NASCIMENTO, R. M. S.; CORREIA, M. G. S. A ingestão de alimentos funcionais e sua contribuição para a diminuição da incidência de doenças. Cadernos de Graduação Ciências Biológicas e da Saúde, v. 1, p. 43-52, 2012.

WOISKY, R. G.; SALATINO, A. Analysis of propolis: some parameters and procedures for chemical quality control. Journal of Apicultural Research, v. 37, n. 2, p. 99-105, 1998. http:// dx.doi.org/10.1080/00218839.1998.11100961.

ZAPATA, L. M.; GERARD, L.; DAVIES, C.; SCHVAB, M. C. Estudio de los componentes antioxidantes y actividad antioxidante en tomates. Ciencia, Docencia y Technologia, v. 35, p. 175-193, 2007. 